\title{
RADIOLOGICAL AND PATHOLOGICAL CORRELATION OF LUNG NODULES IN A BACKGROUND OF METASTATIC DISEASE
}

\author{
AZRA AKHTAR ${ }^{1}$, NOREEN AKHTAR ${ }^{1}$, SAJID MUSHTAQ ${ }^{1}$, USMAN HASSAN ${ }^{1}$, ALI RAZA KHAN ${ }^{2}$ \\ ${ }^{1}$ Department of Pathology, Shaukat Khanum Memorial Cancer Hospital and Research Centre, Lahore, Pakistan, \\ ${ }^{2}$ Department of Surgical Oncology, Shaukat Khanum Memorial Cancer Hospital and Research Centre, Lahore, \\ Pakistan
}

Received: 3 February 2015 / Accepted: 24 April 2015

\begin{abstract}
Background: Computed tomography (CT) imaging has improved the chances of detecting small indeterminate $(<1 \mathrm{~cm})$ lung nodules. The determination of the underlying malignant or benign nature of a lung nodule poses a great diagnostic challenge and depends on a number of factors, including the radiographic appearance of nodule, the presence of non-pulmonary metastases, characteristics of growth and histological criteria.

Methods: The medical records of 89 patients admitted to our specialist cancer centre between 2008 and 2013 were reviewed. Patients of all age groups and tumour category were included in the study. Clinical data of these patients were collected and the following parameters were analysed: Radiographic diagnosis, location, size, laterality and number of nodules and histological impression. The radiological findings were then correlated with histopathological findings. The nodules were sub-classified into groups on the basis of size $(A=0-0.5 \mathrm{~cm} ; \mathrm{B}=0.5-0.9 \mathrm{~cm} ; \mathrm{C}=1.0-1.5 \mathrm{~cm}$ and $\mathrm{D}=>1.5 \mathrm{~cm})$.

Results: CT scan reports of 89 patients with lung nodules were reviewed. On radiology, 73/89 (82\%) were reported to be malignant nodule. Histopathological review of the biopsies of these 89 nodules confirmed malignancy in 50/89 (56.2\%) patients. CT scan was found to be highly sensitive (94\%, $95 \%$ confidence interval [CI]: $83.43-98.68 \%)$ but with a very low specificity $(33.3 \%, 95 \%$ CI: $19.10-50.22 \%)$. CT scan was found to have a higher negative predictive value $(81.2 \%, 95 \%$ CI: $54.34-95.73 \%)$ and a lower positive predictive value 64.4\% (95\% CI: 52.31-75.25\%) when correlated with histopathological findings. Pathology of these nodules included metastatic sarcoma $(27 / 89 ; 30.3 \%)$ and carcinoma $(18 / 89 ; 20.2 \%)$. The frequency of the biopsy-proven malignant nodules on the right side was $26 / 45(57.8 \%)$ and on the left side was $24 / 44(54.5 \%)(P=0.832)$. Malignant nodules were more frequent in lower lobes $(28 / 43,65.1 \%)$ than in upper lobes $(14 / 32,43.8 \%)$. These two sites combined accounted for $84 \%$ of all malignant nodules. There was a significant correlation between nodule size and likelihood of underlying malignancy. The overall prevalence of malignancy in the larger nodules (C and D) was much higher (23/30 and 76.7\%) compared to the smaller sized (A and B) nodules (27/58 and $46.8 \%), P<0.05$.
\end{abstract}

Conclusion: CT scan is a useful tool in the initial clinical assessment of indeterminate lung nodules with high sensitivity (94\%) and a high negative predictive value (81.2\%).

Key words: Computed tomography, fibrosis, indeterminate lung nodule, infection, lung nodule, malignancy, metastases

Correspondence: Dr. Azra Akhter, Department of Pathology, Shaukat Khanum Memorial Cancer Hospital and Research, Centre, Lahore, Pakistan.

Email: azra.akhtar@rocketmail.com

\section{Introduction}

The lung is the most common site for metastasis. The common tumours which metastasise to the lung include 
renal, breast, colon and prostate carcinomas as well as sarcomas. Lung nodules are small, focal and radiographic opacities that can be either single or multiple. Nodules $>3 \mathrm{~cm}$ are called lung masses and can be diagnosed easily on chest X-ray. However, small nodules $(<1 \mathrm{~cm})$, diagnosed only on high resolution computed tomography (CT) scan, are called indeterminate lung nodules. Recent advances in CT imaging have dramatically improved the chances of detecting indeterminate lung nodules.

Determination of small indeterminate lung nodule, or multiple nodules, during the initial evaluation and staging of tumour in a patient, offers a great diagnostic challenge. Malignant or benign nature of pulmonary nodule will affect the treatment plan and the eligibility of the patient for treatment protocols and disease prognosis. The determination or presumption of pulmonary metastasis depends on multiple factors such as radiographic appearance of nodule, presence of evidence of non-pulmonary metastasis, growth characteristics and histological evaluation of biopsy. Nevertheless, radiology alone cannot determine the disease status of a nodule..$^{[1-3]}$

The detection of a solitary pulmonary nodule in healthy adults during evaluation for screening purpose advocates the reliance on growth kinetics. ${ }^{[4,5]}$ The advent of refined CT imaging, particularly helical CT and the ability to obtain thinner axial sections has dramatically increased the detection of pulmonary nodules. ${ }^{[6]}$ Second, evaluation by the picture archive communications system (PACS) allows better enlargement of individual images and less loss of resolution as compared to traditional film images. These advances in radiological techniques allow the detection of small indeterminate nodules that would otherwise have not been possible with the use of plain X-ray technique, thus increasing the likelihood of a CT detected nodule to be malignant. ${ }^{[7-9]}$

To address the challenging question that CT detected lung nodule is more likely to be malignant, the medical records and relevant $\mathrm{CT}$ scans of children and adults who had been referred to our institution for the evaluation of indeterminate lung nodules and lung metastasis were reviewed. The patients' clinical or radiographic characteristics were correlated with histologic features of nodules and disease stage. Most of the nodules diagnosed as malignant on radiology turned out to be benign, proving that histological correlation is an important tool in conjunction with CT scan for confirmation of malignancy in suspicious lung nodules.

\section{Methods}

The clinical data of patients with indeterminate lung nodules detected on a CT scan and referred to Shaukat Khanum Memorial Cancer Hospital and Research Centre (SKMCH and RC), Lahore, Pakistan between 1 January, 2008, and 30 June, 2013, were reviewed. Patients referred for consultation only, and for whom no chest CT was available, were excluded from the study. CT scans showing inflammatory infiltrate or atelectasis were also excluded from our study. Chest CT scans with potential nodules and masses were then reviewed by a single radiologist using PACS to determine the exact number of nodules, the size of the largest nodule or mass, laterality, the pattern of distribution of the nodules and presence of calcifications since calcification can be seen within nodules in case of metastatic osteosarcoma and granulomatous disease. Pulmonary nodules were defined as any round abnormal lung opacities measuring $\leq 3 \mathrm{~cm}$. Lung masses were defined as abnormal, round lung opacities measuring $>3 \mathrm{~cm}$ in any dimension. ${ }^{[10,11]}$ The CT lung nodules were then divided into four groups: Group A with nodules of $5 \mathrm{~mm}$ or less; Group B with nodules between 5 and $9 \mathrm{~mm}$; Group $\mathrm{C}$ with nodules between 10 and $15 \mathrm{~mm}$ and Group D with nodules of $>15 \mathrm{~mm}$. These findings were then correlated with histology. Data were compared using cross-tabulation and associations among factors were examined using the Fischer exact test, $\chi^{2}$ test, Wilcoxon rank sum test or Kruskal-Wallis test as appropriate. Exact statistical tests were used when sample sizes were small. No adjustments were made for multiple comparisons in this exploratory study. $P<0.05$ was considered to be statistically significant. This study was approved by the Institutional Review Board of SKMCH and RC.

\section{Results}

During the study period, a total of 89 patients with lung nodules on CT scan underwent lung biopsies. The biopsy results were correlated with the radiological size of lung nodules including the indeterminate nodules diagnosed on radiology. On radiological impression, a majority 
of these patients $(73 / 89$ and $82.0 \%)$ were reported to have a malignant nodule. In another six patients $(6.7 \%)$, a diagnosis of benign tumour was made, whilst eight patients $(9.0 \%)$ were considered to have indeterminate nodules. Two patients $(2.2 \%)$ with previous follow-up on nodules were reported to have stable nodules. 44 (49.4\%) were on the left side while right-sided nodules were seen in $45(50.6 \%)$ patients. There was no statistical difference in the laterality of the malignant nodules (right $=38 / 45$, $84.4 \%$ and left $=35 / 44,79.5 \% ; P=0.547)$. The relative frequency of nodules was higher in the lower lobes ( $n=43$ and $48.3 \%$ ) than in the upper lobes $(n=32$ and $36.0 \%)$. The remainder of the nodules were reported in the apex (1.1\%), diaphragm (1.1\%), middle lobe (10.1\%) and diffuse/whole lung (2.2\%). A majority of the nodules fell in category B $(31 / 89 ; 34.8 \%)$ followed by A $(21 / 89$, $30.3 \%)$, D (21/89, 23.6\%) and C (9/89, 10.1\%). Table 1 shows the baseline characteristics of lung nodules on radiology in 89 patients.

\section{Table 1: Characteristics of lung nodules in 89 patients}

\begin{tabular}{|c|c|}
\hline Nodule characteristics & Number of patients (\%) \\
\hline \multicolumn{2}{|l|}{ Location } \\
\hline Lower lobe & $43 / 89(48.3)$ \\
\hline Upper lobe & $32 / 89(36.0)$ \\
\hline Middle lobe & $9 / 89(10.1)$ \\
\hline Diaphragm & $1 / 89(1.1)$ \\
\hline Apex & $1 / 89(1.1)$ \\
\hline Others & $3 / 89(3.4)$ \\
\hline \multicolumn{2}{|l|}{ Size } \\
\hline Category A $(\leq 5 \mathrm{~mm})$ & $27 / 89(30.3)$ \\
\hline Category B $(5 \leq 9 \mathrm{~mm})$ & $31 / 89(348)$ \\
\hline Category C $(10 \leq 15 \mathrm{~mm})$ & $9 / 89(10.1)$ \\
\hline Category D (>15 mm) & $21 / 89(23.6)$ \\
\hline \multicolumn{2}{|l|}{ Laterality } \\
\hline Right-sided & $45 / 89(50.6)$ \\
\hline Left-sided & $44 / 89(49.4)$ \\
\hline \multicolumn{2}{|l|}{ Number } \\
\hline One & $20 / 89(22.5)$ \\
\hline Two & $13 / 89(14.6)$ \\
\hline Three & $6 / 89(6.7)$ \\
\hline Multiple (>3) & $50 / 89(56.2)$ \\
\hline \multicolumn{2}{|l|}{ Histopathology } \\
\hline Malignant & $50 / 89(56.2)$ \\
\hline Benign & $39 / 89(43.8)$ \\
\hline
\end{tabular}

Histopathological review of the biopsies on these 89 nodules revealed malignancy in $50(56.2 \%)$ of the patients. The CT scan was found to be highly sensitive (94\%, 95\% confidence interval [CI]: 83.43-98.68\%) but with very low specificity $(33.3 \%$, 95\% CI: $19.10-50.22 \%)$. The positive predictive value of CT scan, when correlated with histopathological findings, was $64.4 \%$ (95\% CI: $52.31-75.25 \%$ ) with a high negative predictive value of $81.2 \%$.

All 50 cases of biopsy-proven malignant nodules were equally likely to have nodules in the right $(26 / 50 ; 52.0 \%)$ or left $(24 / 50 ; 48.0 \%)$ lung $(P=0.832)$. A majority of the malignant nodules were seen in the lower lobe $(28 / 50$, $56.0 \%)$ and upper lobe $(14 / 50,28.0 \%)$. These two sites combined accounted for $84.0 \%$ of all the malignant nodules seen.

There was, however, a significant correlation between nodule size and likelihood of underlying malignancy. The proportion of malignant cases based on size in the order of decreasing percentage was $\mathrm{C}(7 / 9 ; 77.8 \%)$; D (16/21, 76.2\%), A (14/27; 52.8\%) and B (13/31; 41.9\%), $P<0.027$.

The overall prevalence of malignancy in the larger nodules (C, D) was much higher $(23 / 30 ; 76.7 \%)$ compared to the smaller (A, B) nodules $(26 / 58 ; 44.8 \%)$. The proportion of malignancy in nodules increased with increasing number of the nodules $(3>2>1)$. However, overall malignancy rates were lowest in patients with multiple lung nodules. The presence of 1, 2 and 3 nodules on CT scan was associated with $66 \%, 69 \%$ and $83 \%$ risk of malignancy. Table 2 provides details of malignant nodules with increasing number.

Histopathology reports of the lung biopsies showed a variety of diagnoses for the 89 nodules. The most

Table 2: Correlation between the number of nodules and histopathology findings

\begin{tabular}{l|c|c}
\hline Number of nodules & Benign $\boldsymbol{n = 3 9}$ & Malignant $\boldsymbol{n = 5 0}$ \\
\hline One & 7 & 13 \\
\hline Two & 4 & 9 \\
\hline Three & 1 & 5 \\
\hline Multiple $(>3)$ & 27 & 23 \\
\hline
\end{tabular}


commonly reported diagnoses were sarcoma (27/89; $30.3 \%)$ and carcinoma $(18 / 89 ; 20.2 \%)$. The less commonly reported tumours and diagnoses are shown in Table 3. $39 / 89$ (43.8\%) nodules were found to be benign on histopathological evaluation. The most common benign

\section{Table 3: Histopathological classification of lung nodules in 89 patients}

\begin{tabular}{l|c}
\hline Nodule characteristics & $\begin{array}{c}\text { Number of } \\
\text { patients } \boldsymbol{n = 8 9}(\%)\end{array}$ \\
\hline Sarcoma & $27(30.3)$ \\
\hline Carcinoma & $18(20.2)$ \\
\hline Necrosis & $8(9.0)$ \\
\hline Fibrosis & $7(7.9)$ \\
\hline Benign & $4(4.5)$ \\
\hline Haematoma & $4(4.5)$ \\
\hline Silicotic nodule & $4(4.5)$ \\
\hline Anthracotic nodule & $2(2.2)$ \\
\hline Necrotic debris & $2(2.2)$ \\
\hline Germ cell tumour & $2(2.2)$ \\
\hline Lymph node, hilar & $2(2.2)$ \\
\hline Calcification & $1(1.1)$ \\
\hline Granuloma & $3(3.4)$ \\
\hline Haematoma & $1(1.1)$ \\
\hline Non-specific interstitial Pneumonitis & $2(2.2)$ \\
\hline Lung tissue & $1(1.1)$ \\
\hline Wilms' tumour & $1(1.1)$ \\
\hline
\end{tabular}

histological diagnoses were fibrosis $(7 / 39 ; 17.9 \%)$, followed by necrosis $(7 / 39 ; 17.9 \%)$, silicosis $(4 / 39 ; 10.3 \%)$, haematoma $(4 / 39 ; 10.3 \%)$, benign not otherwise classified $(4 / 39 ; 10.3 \%)$, granuloma $(3 / 39 ; 7.7 \%)$, lymph node $(2 / 39$; $5.1 \%)$, debris $(2 / 39,5.1 \%)$, anthracotic nodule $(2 / 39$; $5.1 \%)$, calcification $(1 / 39,2.6 \%)$, non-specific interstitial pneumonitis $(1 / 39 ; 2.6 \%)$, hamartoma $(1 / 39 ; 2.6 \%)$ and benign lung tissue $(1 / 39 ; 2.6 \%)$. These characteristics of benign nodules are described in Tables 4 and 5 .

\section{Discussion}

In this case series of 89 patients with radiographic findings of lung nodules, malignancy was found in a majority of the patients. CT scan was found to be highly sensitive in detecting small indeterminate nodules $(94 \%)$ in patients. However, the positive predictive value of $\mathrm{CT}$ scan was only $64 \%$, due to poor discrimination between benign and malignant nodules on CT scan. Another feature associated with the prediction of malignancy on $\mathrm{CT}$ scan-detected lung nodules was the size of the lung nodule. Given the low positive predictive value, all cases of indeterminate lung nodule should be correlated with biopsy and histopathology.

The majority (56.2\%) of patients with lung nodules in this study had pulmonary metastatic disease, based on clinical or histologic criteria. The overall incidence of

Table 4: Correlation between histopathology and radiographic findings of benign nodules

\begin{tabular}{l|c|c|c|c}
\hline \multirow{2}{*}{ Histopathology finding } & \multicolumn{4}{c}{ Radiographic finding } \\
\cline { 2 - 5 } & Benign & Malignant & Suspicious & Total \\
\hline Fibrosis & 1 & 5 & 0 & 7 \\
\hline Calcification & 0 & 1 & 0 & 1 \\
\hline Debris & 1 & 1 & 0 & 2 \\
\hline Necrosis & 0 & 3 & 4 & 7 \\
\hline Non-specific interstitial pneumonitis & 1 & 0 & 0 & 1 \\
\hline Lymph node & 0 & 0 & 2 & 2 \\
\hline Hamartoma & 0 & 1 & 0 & 1 \\
\hline Silicotic nodule & 1 & 3 & 0 & 4 \\
\hline Lung tissue & 1 & 0 & & 1 \\
\hline Anthracotic nodule & 0 & 2 & & 2 \\
\hline Haematoma & 0 & 4 & 1 & 4 \\
\hline Benign & 0 & 3 & 0 & 4 \\
\hline Granuloma & 0 & 3 & 8 & 3 \\
\hline Total & 5 & 26 & & 39 \\
\hline
\end{tabular}


Table 5: Correlation of size of the nodule with histopathological diagnosis

\begin{tabular}{l|c|c|c}
\hline Radiological size of nodule & Total number of nodules & Biopsy proven malignant & Biopsy proven benign \\
\hline Group A (size 0-5 mm) & 27 & 14 & 13 \\
\hline Group B (size 5-9 mm) & 31 & 12 & 19 \\
\hline Group C (size $10-15 \mathrm{~mm})$ & 9 & 7 & 2 \\
\hline Group D (size $>15 \mathrm{~mm}$ ) & 21 & 16 & 5 \\
\hline
\end{tabular}

pulmonary metastasis in our study group is higher than reported in previous studies of patients with bone and soft tissue sarcomas from other institutions around the world where it ranges from 15 to $26 \%{ }^{[12-15]}$ This is likely because we selected cases that had a previous history of malignancy and we reviewed only those cases where lung nodules had been detected on CT scan and the patients had a histopathological analysis of lung tissue biopsies.

The radiographic characteristics of pulmonary nodules were evaluated using new technologic advances in CT imaging which allowed us to detect multiple small nodules, which would not have been detected otherwise, if older CT techniques had been used. The clinical significance of detecting small pulmonary metastatic lesions has been controversial. Several studies of Wilm's tumour documents nodules detected by CT scan which were not visible on chest X-ray. The effect of this finding on disease prognosis was not consistent. ${ }^{[3,16,17]}$

Several research studies have been done to determine the prognostic significance of the pattern of distribution of metastatic lung nodules in large groups of patients. The results have been variable. However, none of these studies evaluated the size of the lesions. ${ }^{[18-24]}$ The Mayo Clinic CT screening trial showed that $0 \%$ of nodules measuring $<4 \mathrm{~mm}$ and $0.8 \%$ lung nodules measuring between 4 and $7 \mathrm{~mm}$ in diameter were malignant. Another study reported $0 \%$ of nodules measuring $<5 \mathrm{~mm}$ in diameter were malignant, whilst the rate was $5.9 \%$ for those measuring 5-9 $\mathrm{mm}$ in diameter. ${ }^{[25,26]}$ The results of these studies are in contrast to our results since in our study $51.8 \%$ and $31.8 \%$ of category A and B nodules showed malignancy, respectively.

Analysis of our research data also indicated that increasing numbers of nodules are associated with an increased risk of malignancy. The presence of 1,2 and 3 nodules on CT scan was associated with $66 \%, 69 \%$ and $83 \%$ risk of malignancy. The risk of malignancy with an increasing number of nodules is relatively high in our study. Most of the cases with diffuse and bilateral lung nodularity, however, were found to have a benign granulomatous process. This could be explained by the fact that tuberculosis, which is a leading cause of this kind of benign granulomatous process, is endemic in Pakistan.

In addition, the risk of malignancy was also higher in larger nodules compared to smaller nodules. This suggests that risk assignment of lung nodules should be based on the number as well as the size of lung nodules. We did not find any association between laterality and distribution of the lung nodules.

Our study had several limitations. First, we relied on a relatively small sample size from a single institution. Second, the assessment of CT scans was carried out by a single investigator. Third, there is a possibility of sampling errors during a biopsy or attempted resection of pulmonary nodules. This could have been reduced by the use of CTguided needle localisation of a suspicious nodule, but our analyses did not allow us to determine if this technique was used in a systematic manner.

\section{Conclusion}

The classification of indeterminate lung nodules on radiology as malignant or benign depends on a number of factors including their size, laterality, site in the lung and the number of nodules present. We found a significant association of malignancy with larger nodules and an increasing number of nodules. CT scan is a useful tool in the initial clinical assessment of these indeterminate lung nodules and has a high sensitivity of detecting small nodules, with a high negative predictive value, but does require correlation with clinical and histopathological data. 


\section{Conflict of Interest}

The authors declare that they have no conflict of interest.

\section{References}

1. McCarville MB, Lederman HM, Santana VM, et al. Distinguishing benign from malignant pulmonary nodules with helical chest CT in children with malignant solid tumors. Radiology 2006;239:514-20.

2. Picci P, Vanel D, Briccoli A, et al. Computed tomography of pulmonary metastases from osteosarcoma: The less poor technique. A study of 51 patients with histological correlation. Ann Oncol 2001;12:1601-4.

3. Wilimas JA, Douglass EC, Magill HL, et al. Significance of pulmonary computed tomography at diagnosis in Wilms' tumour. J Clin Oncol 1988;6:1144-6.

4. Libby DM, Smith JP, Altorki NK., Managing the small pulmonary nodule discovered by CT. Chest 2004;125: 1522-9.

5. Munden RF, Hess KR. "Ditzels" on chest CT: Survey of members of the society of thoracic radiology. AJR Am J Roentgenol 2001;176:1363-9.

6. Mulshine JL. New developments in lung cancer screening. J Clin Oncol 2005;23:3198-202.

7. Heuvelmans MA, Oudkerk M, de Jong PA, et al. The impact of radiologists' expertise on screen results decisions in a CT lung cancer screening trial. Eur Radiol 2015;25:792-9.

8. Carillo GA, Vázquez JE, Villar AF. Prevalence of benign pulmonary lesions excised for suspicion of malignancy: Could it reflect a quality management index of indeterminate lung lesions? Korean J Thorac Cardiovasc Surg 2014;47:458-64.

9. Gierada DS, Pinsky P, Nath H, et al. Projected outcomes using different nodule sizes to define a positive CT lung cancer screening examination. J Natl Cancer Inst 2014; 106:284.

10. Siegelman SS, Khouri NF, Leo FP, et al. Solitary pulmonary nodules: CT assessment. Radiology 1986;160:307-12.

11. Zerhouni EA, Stitik FP, Siegelman SS, et al. CT of the pulmonary nodule: A cooperative study. Radiology 1986;160: 319-27.

12. Bielack SS, Kempf-Bielack B, Delling G, et al. Prognostic factors in high-grade osteosarcoma of the extremities or trunk: An analysis of 1,702 patients treated on neoadjuvant cooperative osteosarcoma study group protocols. J Clin Oncol 2002;20:776-90.

13. Kaste SC, Pratt CB, Cain AM, et al. Metastases detected at the time of diagnosis of primary pediatric extremity osteosarcoma at diagnosis: Imaging features. Cancer 1999;86: 1602-8.

14. Pappo AS, Rao BN, Jenkins JJ, et al. Metastatic non rhabdomyosarcomatous soft-tissue negative predictive value (81.2\%) experience. Med Pediatr Oncol 1999;33:76-82.

15. Tsuchiya H, Kanazawa Y, Abdel-Wanis ME, et al. Effect of timing of pulmonary metastases identification on prognosis of patients with osteosarcoma: The Japanese musculoskeletal oncology group study. J Clin Oncol 2002;20: 3470-7.

16. Green DM, Fernbach DJ, Norkool P, et al. The treatment of Wilms' tumour patients with pulmonary metastases detected only with computed tomography: A report from the national Wilms' tumour study. J Clin Oncol 1991;9:1776-81.

17. Owens CM, Veys PA, Pritchard J, et al. Role of chest computed tomography at diagnosis in the management of Wilms' tumor: A study by the United Kingdom children's cancer study group. J Clin Oncol 2002;20:2768-73.

18. Daw NC, Billups CA, Rodriguez-Galindo C, et al. Metastatic osteosarcoma. Cancer 2006;106:403-12.

19. Harris MB, Gieser P, Goorin AM, et al. Treatment of metastatic osteosarcoma at diagnosis: A Pediatric oncology group study. J Clin Oncol 1998;16:3641-8.

20. Kager L, Zoubek A, Pötschger U, et al. Primary metastatic osteosarcoma: Presentation and outcome of patients treated on neoadjuvant cooperative osteosarcoma study group protocols. J Clin Oncol 2003;21:2011-8.

21. Meyer WH, Schell MJ, Kumar AP, et al. Thoracotomy for pulmonary metastatic osteosarcoma. An analysis of prognostic indicators of survival. Cancer 1987;59:374-9.

22. Meyers PA, Heller G, Healey JH, et al. Osteogenic sarcoma with clinically detectable metastasis at initial presentation. J Clin Oncol 1993;11:449-53.

23. Putnam JB Jr., Roth JA, Wesley MN, et al. Survival following aggressive resection of pulmonary metastases from osteogenic sarcoma: Analysis of prognostic factors. Ann Thorac Surg 1983;36:516-23.

24. Roth JA, Putnam JB Jr., Wesley MN, et al. Differing determinants of prognosis following resection of pulmonary metastases from osteogenic and soft tissue sarcoma patients. Cancer 1985;55:1361-6.

25. Swensen SJ, Jett JR, Sloan JA, et al. Screening for lung cancer with low-dose spiral computed tomography. Am J Respir Crit Care Med 2002;165:508-13.

26. Henschke CI, Yankelevitz DF, Naidich DP, et al. CT screening for lung cancer: Suspiciousness of nodules according to size on baseline scans. Radiology 2004;231:164-8. 